\title{
The Exponentiated Weibull-Pareto Distribution with Application
}

\author{
Ahmed Z. Afify and Haitham M. Yousof \\ Department of Statistics, Mathematics and Insurance \\ Benha University, Egypt \\ G. G. Hamedani \\ Department of Mathematics, Statistics and Computer Science \\ Marquette University, USA \\ Gokarna R. Aryal \\ Department of Mathematics, Statistics and Computer Science \\ Purdue University Northwest, USA
}

Received 5 August 2015

Accepted 28 February 2016

\begin{abstract}
A new generalization of the Weibull-Pareto distribution called the exponentiated Weibull-Pareto distribution is defined and studied. Various structural properties including ordinary moments, quantiles, Rényi and q-entropies and order statistics are derived. We proposed the method of maximum likelihood for estimating the model parameters. We provide the simulation results to assess the performance of the proposed model. The usefulness and flexibility of the new model is illustrated using real data.

Keywords: Exponentiated distribution, Weibull-Pareto distribution, Rényi entropy, Order statistics, Parameter estimation
\end{abstract}

2000 Mathematics Subject Classification: 60E05, 62P99

\section{Introduction}

Statistical distributions are very useful in describing and predicting real world phenomena. In fact, the statistics literature is filled with hundreds of continuous univariate distributions and their successful applications. The Weibull distribution is one of the most commonly used lifetime distribution in modeling lifetime data. In practice, it has been shown to be very flexible in modeling various types of lifetime distributions with monotone failure rates but it is not useful for modeling the bathtub shaped and the unimodal failure rates which are common in reliability and biological studies. To overcome this shortcoming, several generalizations of the classical Weibull distribution have been discussed by different authors. Some notable generalizations include the generalized Weibull distribution by Mudholkar and Kollia [27], the exponentiated-Weibull distribution by Mudholkar et al. [28], the beta-Weibull distribution by Famoye et al. [13], the Kumaraswamy Weibull by Cordeiro 
et al. [12], the transmuted Weibull distribution by Aryal et al. [8], the Weibull Lomax distribution by Tahir et al. [32] and the transmuted Weibull Lomax by Afify et al. [3].

Similarly, the Pareto distribution is well known in the literature for its capability in modeling the heavy-tailed distributions. Applications of Pareto distribution have appeared in several areas including reliability, exponential tilting (weighting) in finance and actuarial sciences, as well as in economics. A hierarchy of the Pareto distributions has been established starting from the classical Pareto (I) to Pareto (IV) distributions with subsequent additional parameters related to location, shape and inequality. To add flexibility to the Pareto distribution various generalizations of Pareto distribution have appeared in the literature. For example, Alzaatreh et al. [5] proposed the WeibullPareto, Bourguignon et al. [11] introduced the Kumaraswamy-Pareto, Kareema et al. [23] defined the exponentiated-Pareto, Alzaatreh et al. [6] introduced the Gamma-Pareto, Akinsete et al. [4] introduced the beta-Pareto distributions, Zea et al. [34] studied the beta exponentiated Pareto, Mahmoudi [26] proposed the beta generalized Pareto. Recently, a generator of distributions called the Weibull-G class was proposed and studied by Bourguignon et al. [10]. Tahir et al. [33] used this formulation to develop the Weibull-Pareto (WP) distribution. The WP distribution has higher skewness as compared to a Weibull distribution and therefore is more suitable to model a heavily skewed data which commonly arise in reliability and survival analysis.

The cumulative distribution function (cdf) (for $x>\theta>0$ ) of the Weibull-Pareto (WP) distribution as proposed in Tahir et al. [33] is given by

$$
G(x ; a, b, \theta)=1-\exp \left\{-\left[\left(\frac{x}{\theta}\right)^{a}-1\right]^{b}\right\}
$$

where $\theta$ is a scale parameter, $a>0$ and $b>0$ are shape parameters. The corresponding probability density function (pdf) is given by

$$
g(x ; a, b, \theta)=\frac{a b}{\theta^{a}} x^{a-1}\left[\left(\frac{x}{\theta}\right)^{a}-1\right]^{b-1} \exp \left\{-\left[\left(\frac{x}{\theta}\right)^{a}-1\right]^{b}\right\} .
$$

In this article we would like to provide an extension of the WP distribution using the exponentiated generalization, say $G^{\alpha}$. The new model is referred to as the exponentiated Weibull-Pareto (EWP) distribution.

Definition: Let $G$ be the cdf of and absolutely continuous random variable with support on $(a, b)$ where the interval may be unbounded, and let $\alpha$ be a positive real number. The random variable $X$ has a $G^{\alpha}$ distribution if its cdf, denoted by $F(x)$, is given by

$$
F(x)=[G(x)]^{\alpha}
$$

Note that this is the $\alpha$ th power of the base line distribution function $G(x)$, and the corresponding pdf of $X$ is given by

$$
f(x)=\alpha g(x)[G(x)]^{\alpha-1} .
$$

The class of $G^{\alpha}$ distributions contains certain well-known distributions for which their cdf's have closed forms, see for example, Gupta and Kundu [17-20] and Nadarajah [29]. Shakil and 
Ahsanullah [31] introduced some distributional properties of order statistics and record values from $G^{\alpha}$ distributions.

Recently, many authors constructed generalizations based on the exponentiation method. For instance, Afify et al. [2] studied the exponentiated transmuted generalized Rayleigh, Huang and Oluyede [22] introduced exponentiated Kumaraswamy-Dagum, Nadarajah and Kotz pioneered the exponentiated gamma, exponentiated Fréchet and exponentiated Gumbel, Gupta et al. [16] proposed the exponentiated exponential distributions. It can be seen from the above mentioned articles that the exponentiated models are significantly more flexible than their classical counterparts to characterize the real life phenomenons. The goal of the present work is to propose and study a generalization of the Weibull-Pareto distribution via the exponentiated method describe above in order to obtain a larger class of flexible parametric models. The rest of the article is outlined as follows. In Section 2, we define the EWP distribution and provide the graphical presentation for its pdf and hazard rate function (hrf). A useful mixture representation for its pdf and cdf is provided in Section 3. Section 4 provides the mathematical properties including quantile function, moments and Rényi q-entropies. Some characterization results are provided in Section 5. In Section 6, the order statistics and their moments are discussed. The maximum likelihood estimates (MLEs) for the model parameters are demonstrated in Section 7. In Section 8, simulation results to assess the performance of the proposed maximum likelihood estimation procedure are discussed. In section 9, the EWP distribution is applied to a real data set to illustrate its usefulness.

\section{The Exponentiated Weibull-Pareto Distribution}

A random variable $X$ is said to have an exponentiated Weibull-Pareto (EWP) distribution, denoted by $X \sim \operatorname{EWP}(x, a, b, \theta, \alpha)$, if its cdf is given (for $x>\theta$ ) by

$$
F(x)=\left(1-\exp \left\{-\left[\left(\frac{x}{\theta}\right)^{a}-1\right]^{b}\right\}\right)^{\alpha}
$$

The corresponding pdf of $X$ is

$$
f(x)=\frac{a b \alpha}{\theta^{a}} x^{a-1}\left[\left(\frac{x}{\theta}\right)^{a}-1\right]^{b-1} \exp \left\{-\left[\left(\frac{x}{\theta}\right)^{a}-1\right]^{b}\right\}\left(1-\exp \left\{-\left[\left(\frac{x}{\theta}\right)^{a}-1\right]^{b}\right\}\right)^{\alpha-1}
$$

where $\theta>0$ is a scale parameter representing the characteristic life. Also, $a, b$ and $\alpha$ are positive shape parameters representing the different patterns of the EWP distribution.

Figure 1 provides some plots of the EWP density curves for selected values of the parameters $a$, $b, \theta$ and $\alpha$. 

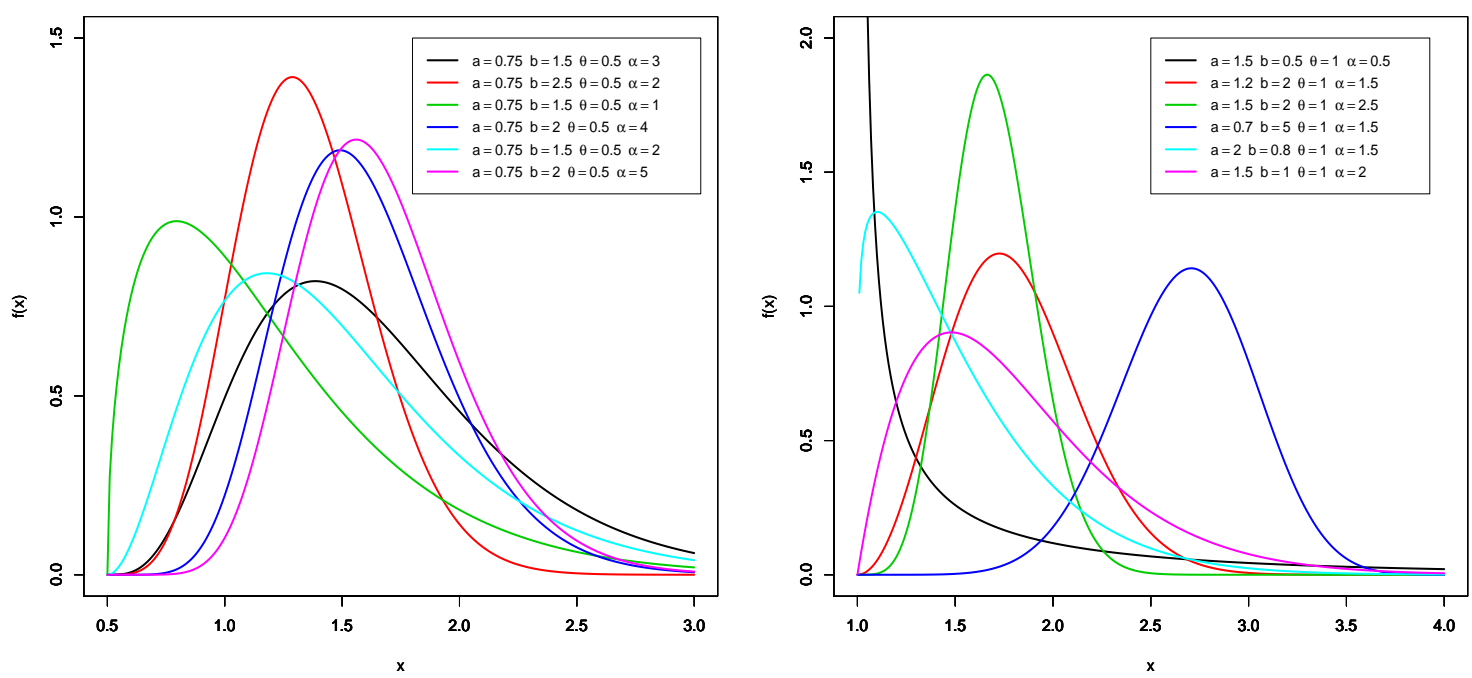

Fig. 1. pdf of EWP distribution for selected values of the parameters

The reliability function $(R(x))$, the hazard rate function $(h(x))$, the reversed-hazard rate function $(r(x))$ and cumulative hazard rate function $(H(x))$ of $X$ are, respectively, given by

$$
\begin{aligned}
& R(x)=1-\left[1-\exp \left\{-\left[\left(\frac{x}{\theta}\right)^{a}-1\right]^{b}\right\}\right]^{\alpha}, \\
& h(x)=\frac{a b \alpha x^{a-1}\left[\left(\frac{x}{\theta}\right)^{a}-1\right]^{b-1} \exp \left\{-\left[\left(\frac{x}{\theta}\right)^{a}-1\right]^{b}\right\}\left[1-\exp \left(-\left[\left(\frac{x}{\theta}\right)^{a}-1\right]^{b}\right)\right]^{\alpha-1}}{\theta^{a}\left[1-\left(1-\exp \left\{-\left[\left(\frac{x}{\theta}\right)^{a}-1\right]^{b}\right\}\right)^{\alpha}\right]}, \\
& r(x)=\frac{a b \alpha x^{a-1}\left[\left(\frac{x}{\theta}\right)^{a}-1\right]^{b-1} \exp \left\{-\left[\left(\frac{x}{\theta}\right)^{a}-1\right]^{b}\right\}}{\theta^{a}\left[1-\exp \left\{-\left[\left(\frac{x}{\theta}\right)^{a}-1\right]^{b}\right\}\right]}
\end{aligned}
$$

and

$$
H(x)=-\ln \left\{1-\left(1-\exp \left\{-\left[\left(\frac{x}{\theta}\right)^{a}-1\right]^{b}\right\}\right)^{\alpha}\right\} .
$$

Plots of the hazard rate function of EWP for selected values of the parameters $a, b, \theta$ and $\alpha$ are given in Figure 2. It can be seen that the hazard rate function is very flexible so the proposed distribution should be useful to model increasing, decreasing and constant failure rates behavior. 

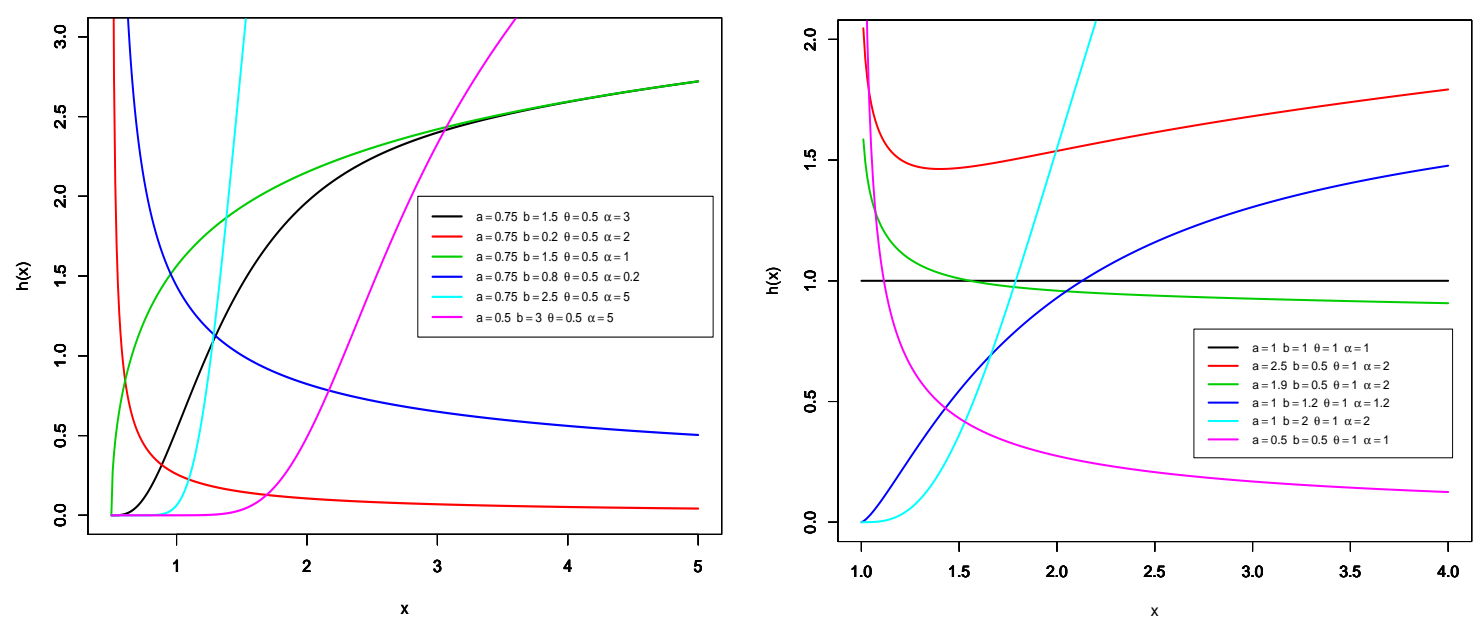

Fig. 2. Hazard rate function of EWP distribution for selected values of the parameters

\section{Mixture Representation}

As presented in Bourguignon et al. [10], Weibull-G class can be expressed in terms of odds ratio $G(x) /[1-G(x)]$ of the base distribution. The EWP density function given in (2.2) can be expressed as

$$
\begin{aligned}
f(x)= & b \alpha g(x) \frac{G(x)^{b-1}}{\bar{G}(x)^{b+1}} \sum_{i=0}^{\infty} \frac{(-1)^{i} \Gamma(\alpha)}{i ! \Gamma(\alpha-i)} \exp \left\{-(i+1)\left(\frac{G(x)}{\bar{G}(x)}\right)^{b}\right\} \\
= & b \alpha\left(\frac{a}{x}\right)\left(\frac{x}{\theta}\right)^{-a} \frac{\left[1-\left(\frac{x}{\theta}\right)^{-a}\right]^{b-1}}{\left\{1-\left[1-\left(1+\frac{x}{\theta}\right)^{-a}\right]\right\}^{b+1}} \\
& \times \sum_{i=0}^{\infty} \frac{(-1)^{i} \Gamma(\alpha)}{i ! \Gamma(\alpha-i)} \exp \left[-(i+1)\left(\frac{\left[1-\left(\frac{x}{\theta}\right)^{-a}\right]}{1-\left[1-\left(\frac{x}{\theta}\right)^{-a}\right]}\right)^{b}\right] .
\end{aligned}
$$

Using the power series expansion, we have

$$
\exp \left[-(i+1)\left(\frac{\left[1-\left(\frac{x}{\theta}\right)^{-a}\right]}{1-\left[1-\left(\frac{x}{\theta}\right)^{-a}\right]}\right)^{b}\right]=\sum_{k=0}^{\infty} \frac{(-1)^{k}(i+1)^{k}}{k !} \frac{\left[1-\left(\frac{x}{\theta}\right)^{-a}\right]^{k b}}{\left\{1-\left[1-\left(\frac{x}{\theta}\right)^{-a}\right]\right\}^{k b}}
$$


Substituting in (3.1) and after some calculations we obtain

$$
\begin{aligned}
f(x)= & \frac{a b \alpha}{\theta}\left(\frac{a}{x}\right)\left(\frac{x}{\theta}\right)^{-a} \sum_{i, k=0}^{\infty} \frac{(-1)^{i+k} \Gamma(\alpha)(i+1)^{k}}{i ! k ! \Gamma(\alpha-i)} \\
& \times\left[1-\left(1+\frac{x}{\theta}\right)^{-a}\right]^{k b+b-1}\left\{1-\left[1-\left(\frac{x}{\theta}\right)^{-a}\right]\right\}^{-k b-b-1} .
\end{aligned}
$$

After a power series expansion, we get

$$
\begin{aligned}
f(x)= & \sum_{j, i, k=0}^{\infty} \frac{(-1)^{i+k} \Gamma(\alpha)(i+1)^{k} \alpha b}{j ! i ! k ! \Gamma(\alpha-i)[(k+1) b+j]} \frac{\Gamma((k+1) b+j+1)}{\Gamma((k+1) b+1)} \\
& \times[(k+1) b+j]\left(\frac{a}{x}\right)\left(\frac{x}{\theta}\right)^{-a}\left\{1-\left(\frac{x}{\theta}\right)^{-a}\right\}^{(k+1) b+j-1}
\end{aligned}
$$

Equation (3.2) can be rewritten as follows

$$
f(x ; a, b, \alpha, \theta)=\sum_{j, k=0}^{\infty} v_{j, k} h(x ; a, \theta,(k+1) b+j)
$$

where

$$
v_{j, k}=\sum_{i=0}^{\infty} \frac{(-1)^{i+k} \Gamma(\alpha)(i+1)^{k} \alpha b}{j ! i ! k ! \Gamma(\alpha-i)[(k+1) b+j]} \frac{\Gamma((k+1) b+j+1)}{\Gamma((k+1) b+1)}
$$

and $h(x ; a, \theta,(k+1) b+j)$ is the density of exponentiated-Pareto (EP) with parameters $a, \theta$ and $(k+1) b+j$. This means that the EWP density can be expressed as a mixture of EP densities. So, several of its properties can be derived from those of the EP model.

Similarly, the cdf of the EWP in (2.1) can be expressed in the same mixture form as follows

$$
F(x ; a, b, \theta, \alpha)=\sum_{j, k=0}^{\infty} v_{j, k} H(x ; a, \theta,(k+1) b+j),
$$

where $H(x ; a, \theta,(k+1) b+j)=\left[1-\left(\frac{x}{\theta}\right)^{-a}\right]^{(k+1) b+j}$ is the cdf of EP with parameters $a, \theta$ and $(k+1) b+j$.

\section{Mathematical Properties}

Established algebraic expansions to determine some structural properties of the EWP distribution can be more efficient than computing those directly by numerical integration of its density function. The statistical properties of the EWP distribution including quantile function, ordinary moments and Rényi and q-entropies are provided in this section.

\subsection{Quantile Function}

The quantile function (qf) of $X$, where $X \sim E W P(a, b, \theta, \alpha)$, is obtained by inverting (2.1) as 


$$
Q(u)=\theta\left\{1+\left[-\ln \left(1-u^{\frac{1}{\alpha}}\right)\right]^{\frac{1}{b}}\right\}^{\frac{1}{a}}, 0 \leq u \leq 1 .
$$

Simulating the EWP random variable is straightforward. If $U$ is a uniform variate on the unit interval $(0,1)$, then the random variable $X=Q(U)$ follows (2.1), i.e. $X \sim \operatorname{EWP}(a, b, \theta, \alpha)$.

\subsection{Moments}

The $r$ th order moment $\left(E\left(X^{r}\right)\right)$, denoted by $\mu_{r}^{\prime}$, of the $\operatorname{EWP}(x, a, b, \theta, \alpha)$ is given by

$$
\mu_{r}^{\prime}=\sum_{j, k=0}^{\infty} v_{j, k} \int_{0}^{\infty} x^{r} h(x, a, \theta,(k+1) b+j) d x .
$$

Therefore, for $r \leq \alpha$ we have

$$
\mu_{r}^{\prime}=\theta^{r} \sum_{j, k=0}^{\infty} v_{j, k}[(k+1) b+j] B\left(\frac{a-r}{\alpha},[(k+1) b+j]\right),
$$

where $B(m, n)=\int_{0}^{1} t^{m-1}(1-t)^{n-1} d t$ is the beta function.

In particular, for $r=1$ in (4.1), we obtain the mean of $X$ as follows

$$
\mu_{1}^{\prime}=\theta \sum_{j, k=0}^{\infty} v_{j, k}[(k+1) b+j] B\left(\frac{a-1}{a},[(k+1) b+j]\right) .
$$

The variance, skewness, and kurtosis can be calculated from the ordinary moments using wellknown relationships.

Using the relation between the central moments and non-centeral moments, we can obtain the $n$th central moments, denoted by $M_{n}$, of a EWP random variable as

$$
\begin{aligned}
M_{n} & =E\left(X-\mu_{1}^{\prime}\right)^{n}=\sum_{r=0}^{n}\left(\begin{array}{l}
n \\
r
\end{array}\right)\left(-\mu_{1}^{\prime}\right)^{n-r} E\left(X^{r}\right) \\
& =\sum_{r=0}^{n}\left(\begin{array}{l}
n \\
r
\end{array}\right)(-1)^{n-r}\left(\mu_{1}^{\prime}\right)^{n-r} \mu_{r}^{\prime}
\end{aligned}
$$

and the cumulants $\left(\kappa_{n}\right)$ of $X$ are obtained from (4.1) as

$$
\kappa_{n}=\mu_{n}^{\prime}-\sum_{r=0}^{n-1}\left(\begin{array}{l}
n-1 \\
r-1
\end{array}\right)\left(\kappa_{r}\right)\left(\mu_{n-r}^{\prime}\right)
$$

where $\kappa_{1}=\mu_{1}^{\prime}$. Hence, $\kappa_{2}=\mu_{2}^{\prime}-\mu_{1}^{\prime 2}, \kappa_{3}=\mu_{3}^{\prime}-3 \mu_{2}^{\prime} \mu_{1}^{\prime}+\mu_{1}^{\prime 3}$ etc. The skewness and kurtosis measures can be calculated from the ordinary moments using well-known relationships.

The $r$ th descending factorial moment of $X$ is

$$
\mu_{(r)}^{\prime}=E\left[X^{(r)}\right]=E[X(X-1) \times \ldots \times(X-r+1)]=\sum_{n=0}^{r} s(r, n) \mu_{n}^{\prime},
$$

where $s(r, n)=(n !)^{-1}\left[d^{n} x^{(r)} / d x^{n}\right]_{x=0}$ is the Stirling number of the first kind. 


\subsection{Rényi and q-Entropies}

The Rényi entropy of a random variable $X$ represents a measure of variation of the uncertainty and is defined by

$$
I_{\delta}(X)=\frac{1}{1-\delta} \log \int_{-\infty}^{\infty} f^{\delta}(x) d x, \delta>0 \text { and } \delta \neq 1 .
$$

For EWP distribution the Rényi entropy is derived as below. We have

$$
f^{\delta}(x)=\sum_{i=0}^{\infty} \frac{(-1)^{i} \Gamma(\delta(\alpha-1)+1) \alpha^{\theta}}{i ! \Gamma(\delta(\alpha-1)+1-i)} b^{\theta} g(x)^{\delta} \frac{G(x)^{\delta(b-1)}}{\bar{G}(x)^{\delta(b+1)}} \exp \left\{-(\delta+i)\left[\frac{G(x)}{\bar{G}(x)}\right]^{b}\right\} .
$$

Using a power series for the exponential function and the generalized binomial expansion in the above result, we obtain

$$
f^{\delta}(x)=\sum_{j, k=0}^{\infty} \xi_{j, k} g(x)^{\delta} G(x)^{k b+\delta(b-1)+j}
$$

where

$$
\xi_{j, k}=\sum_{i=0}^{\infty} \frac{(-1)^{i+k} \Gamma(\delta(\alpha-1)+1) \Gamma(b(k+\delta)+\delta+j)(i+\delta)^{k} \alpha^{\theta}}{j ! i ! k ! \Gamma(\delta(\alpha-1)+1-i) \Gamma(b(k+\delta)+\delta)} .
$$

For $\delta(\alpha+1)>1$ and $\delta(b-1)>-1$ transforming variables and integrating, we get

$$
I_{\delta}(X)=\frac{1}{1-\delta} \log \left\{\sum_{j, k=0}^{\infty} \xi_{j, k} \frac{\theta(\alpha \theta)^{\delta}}{\alpha} B\left(\frac{\delta(\alpha+1)-1}{\alpha}, \rho\right)\right\},
$$

where $\rho=b k+\delta(b-1)+j+1$.

The q-entropy, say $H_{q}(X)$, is defined by

$$
H_{q}(X)=\frac{1}{q-1} \log \left\{1-\int_{-\infty}^{\infty} f^{q}(x) d x\right\}, q>0 \text { and } q \neq 1 .
$$

Then, for $q(\alpha+1)>1$ and $q(b-1)>-1$, we cam write

$$
H_{q}(X)=\frac{1}{q-1} \log \left\{1-\left[\sum_{j, k=0}^{\infty} \xi_{j, k}^{*} \frac{\theta(\alpha \theta)^{q}}{\alpha} B\left(\frac{q(\alpha+1)-1}{\alpha}, \rho^{*}\right)\right]\right\},
$$

where $\rho^{*}=b k+q(b-1)+j+1$ and

$$
\xi_{j, k}^{*}=\sum_{i=0}^{\infty} \frac{(-1)^{i+k} \Gamma(q(\alpha-1)+1) \Gamma(b(k+q)+q+j)(i+q)^{k} \alpha^{\theta}}{j ! i ! k ! \Gamma(q(\alpha-1)+1-i) \Gamma(b(k+q)+q)} .
$$

\section{Characterizations}

This section deals with various characterizations of EWP distribution. These characterizations are based on: $(i)$ a simple relationship between two truncated moments; (ii) the hazard function and (iii) truncated moment of certain function of the random variable. Our first characterization result 
employs a theorem due to Glänzel [14], see Theorem 1 below. Note that the result holds also when the interval $H$ is not closed. Moreover it could be also applied when the cdf $F$ does not have a closed form. As shown in Glänzel [15] this characterization is stable in the sense of weak convergence.

Theorem 1. Let $(\Omega, \mathscr{F}, \mathbf{P})$ be a given probability space and let $H=[d, e]$ be an interval for some $d<e(d=-\infty, e=\infty$ might as well be allowed). Let $X: \Omega \rightarrow H$ be a continuous random variable with the distribution function $F$ and let $g$ and $h$ be two real functions defined on $H$ such that

$$
\mathbf{E}[g(X) \mid X \geq x]=\mathbf{E}[h(X) \mid X \geq x] \eta(x), \quad x \in H,
$$

is defined with some real function $\eta$. Assume that $g, h \in C^{1}(H), \eta \in C^{2}(H)$ and $F$ is twice continuously differentiable and strictly monotone function on the set $H$. Finally, assume that the equation $h \eta=g$ has no real solution in the interior of $H$. Then $F$ is uniquely determined by the functions $g$, $h$ and $\eta$, particularly

$$
F(x)=\int_{d}^{x} C\left|\frac{\eta^{\prime}(u)}{\eta(u) h(u)-g(u)}\right| \exp (-s(u)) d u,
$$

where the function $s$ is a solution of the differential equation $s^{\prime}=\frac{\eta^{\prime} h}{\eta h-g}$ and $C$ is the normalization constant, such that $\int_{H} d F=1$.

\subsection{Characterization based on truncated moments}

The first characterization of EWP is given in proposition 5.1 and it is based on a simple relationship between two truncated moments.

Proposition 5.1. Let $X: \Omega \rightarrow(\theta, \infty)$ be a continuous random variable and let $h(x)=$ $\left\{1-\exp \left(-\left[\left(\frac{x}{\theta}\right)^{a}-1\right]^{b}\right)\right\}^{1-\alpha}$ and $g(x)=h(x) \exp \left(-\left[\left(\frac{x}{\theta}\right)^{a}-1\right]^{b}\right)$ for $x>\theta$. The random variable $X$ belongs to EWP family (2.2) if and only if the function $\eta$ defined in Theorem 1 has the form

$$
\eta(x)=\frac{1}{2} \exp \left(-\left[\left(\frac{x}{\theta}\right)^{a}-1\right]^{b}\right), x>\theta .
$$

Proof: Let $X$ be a random variable with pdf (2.2), then

$$
(1-F(x)) E[h(x) \mid X \geq x]=\alpha \exp \left(-\left[\left(\frac{x}{\theta}\right)^{a}-1\right]^{b}\right), \quad x>\theta,
$$

and

$$
(1-F(x)) E[g(x) \mid X \geq x]=\frac{\alpha}{2} \exp \left(-\left[\left(\frac{x}{\theta}\right)^{a}-1\right]^{b}\right), \quad x>\theta,
$$

and finally

$$
\eta(x) h(x)-g(x)=\frac{1}{2} h(x)\left\{-\exp \left(-\left[\left(\frac{x}{\theta}\right)^{a}-1\right]^{b}\right)\right\}<0 \text { for } x>\theta .
$$


Conversely, if $\eta$ is given as above, then

$$
s^{\prime}(x)=\frac{\eta^{\prime}(x) h(x)}{\eta(x) h(x)-g(x)}=a b \theta^{-a} x^{a-1}\left[\left(\frac{x}{\theta}\right)^{a}-1\right]^{b-1}, \quad x>\theta
$$

and hence

$$
s(x)=\left[\left(\frac{x}{\theta}\right)^{a}-1\right]^{b}, \quad x>\theta .
$$

Now, in view of Theorem $1, X$ has density (2.2).

Corollary 5.1. Let $X: \Omega \rightarrow(\theta, \infty)$ be a continuous random variable and let $h(x)$ be as in Proposition 5.1. The pdf of $X$ is (2.2) if and only if there exist functions $g$ and $\eta$ defined in Theorem 1 satisfying the differential equation

$$
\frac{\eta^{\prime}(x) h(x)}{\eta(x) h(x)-g(x)}=a b \theta^{-a} x^{a-1}\left[\left(\frac{x}{\theta}\right)^{a}-1\right]^{b-1}, \quad x>\theta .
$$

Note that the general solution of the differential equation in Corollary 5.1 is

$$
\eta(x)=\exp \left(\left[\left(\frac{x}{\theta}\right)^{a}-1\right]^{b}\right)\left[\begin{array}{c}
-\int a b \theta^{-a} x^{a-1}\left[\left(\frac{x}{\theta}\right)^{a}-1\right]^{b-1} \times \\
\exp \left(-\left[\left(\frac{x}{\theta}\right)^{a}-1\right]^{b}\right)(h(x))^{-1} g(x) d x+D
\end{array}\right],
$$

where $D$ is a constant. Note that a set of functions satisfying the differential equation (5.1) is given in Proposition 5.1 with $D=0$. However, it should be also noted that there are other triplets $(h, g, \eta)$ satisfying the conditions of Theorem 1 .

\subsection{Characterization based on hazard function}

It is known that the hazard function, $h_{F}$, of a twice differentiable distribution function, $F$, satisfies the first order differential equation

$$
\frac{f^{\prime}(x)}{f(x)}=\frac{h_{F}^{\prime}(x)}{h_{F}(x)}-h_{F}(x) .
$$

For many univariate continuous distributions, this is the only characterization available in terms of the hazard function. The following characterization establish a non-trivial characterization for EWP distribution in terms of the hazard function when $\alpha=1$, which is not of the trivial form given in (5.2).

Proposition 5.2. Let $X: \Omega \rightarrow(\theta, \infty)$ be a continuous random variable. Then for $\alpha=1$, the pdf of $X$ is (2.2) if and only if its hazard function $h_{F}(x)$ satisfies the differential equation

$$
h_{F}^{\prime}(x)-(a-1) x^{-1} h_{F}(x)=a^{2} b(b-1) \theta^{-2 a} x^{2(a-1)}\left[\left(\frac{x}{\theta}\right)^{a}-1\right]^{b-2}
$$

with the boundary condition $\lim _{x \rightarrow \theta^{-}} h_{F}(x)=0$ for $b>1$. 
Proof. If $X$ has pdf (2.2), then clearly (5.3) holds. On the other hand, if (5.3) holds, then

$$
\frac{d}{d x}\left\{x^{-(a-1)} h_{F}(x)\right\}=a b \theta^{-a} \frac{d}{d x}\left\{x^{2(a-1)}\left[\left(\frac{x}{\theta}\right)^{a}-1\right]^{b-1}\right\},
$$

or, equivalently,

$$
h_{F}(x)=a b \theta^{-a} x^{(a-1)}\left[\left(\frac{x}{\theta}\right)^{a}-1\right]^{b-1},
$$

which is the hazard function of the EWP distribution.

\subsection{Characterization based on truncated moment of certain function of the random variable}

In this subsection we present the characterizations based on truncated moment of certain function of the random variable. The following propositions are based on the technical report by Hamedani [21] which can be used to characterize EWP distribution.

Proposition 5.3. Let $X: \Omega \rightarrow(a, b)$ be a continuous random variable with cdf $F$. Let $\psi(x)$ be a differentiable function on $(a, b)$ with $\lim _{x \rightarrow a^{+}} \psi(x)=1$. Then for $\delta \neq 1$,

$$
E[\psi(X) \mid X \geq x]=\delta \psi(x), \quad x \in(a, b),
$$

if and only if

$$
\psi(x)=(1-F(x))^{\frac{1}{\delta}-1}, \quad x \in(a, b) .
$$

Proposition 5.4. Let $X: \Omega \rightarrow(a, b)$ be a continuous random variable with cdf $F$. Let $\psi_{1}(x)$ be a differentiable function on $(a, b)$ with $\lim _{x \rightarrow b} \psi_{1}(x)=1$. Then for $\delta_{1} \neq 1$,

$$
E\left[\psi_{1}(X) \mid X \leq x\right]=\delta_{1} \psi_{1}(x), \quad x \in(a, b),
$$

if and only if

$$
\psi_{1}(x)=(F(x))^{\frac{1}{\delta_{1}}-1}, \quad x \in(a, b) .
$$

Proposition 5.5. Let $X: \Omega \rightarrow(a, b)$ be a continuous random variable and let $\psi \in C^{1}(a, b)$ and $\varphi \in C^{2}(a, b)$ such that $\lim _{x \rightarrow a^{-}}\left(\int_{a}^{x} \frac{\varphi^{\prime}(u)}{\varphi(u)-\psi(u)} d u\right)=\infty$. Then

$$
E[\psi(X) \mid X \geq x]=\varphi(x)
$$

implies

$$
1-F(x)=\exp \left\{-\int_{a}^{x} \frac{\varphi^{\prime}(u)}{\varphi(u)-\psi(u)} d u\right\} .
$$

Remark: It is easy to see that for certain functions $\psi(x)$ on $(\theta, \infty) ;(a)$ Proposition 5.4 provides a characterization of EWP distribution; (b) Propositions 5.3 and 5.5 provide characterizations of EWP distribution for $\alpha=1$. 


\section{Order Statistics}

If $X_{1}, X_{2}, \ldots, X_{n}$ is a random sample of size $n$ from the EWP distribution and $X_{1: n}, X_{2: n}, \ldots, X_{n: n}$ be the corresponding order statistics. Then the pdf of $i$ th order statistics $\left(X_{i: n}\right)$ is denoted by $f_{i: n}(x)$ and is given by

$$
f_{i: n}(x)=\frac{f(x)}{\mathrm{B}(i, n-i+1)} \sum_{j=0}^{n-1}(-1)^{j}\left(\begin{array}{c}
n-1 \\
j
\end{array}\right) F^{i+j-1}(x) .
$$

Note that

$$
F^{i+j-1}(x)=\sum_{m=0}^{\infty}(-1)^{m}\left(\begin{array}{c}
\alpha(i+j-1) \\
m
\end{array}\right) \exp \left\{-m\left\{\left(1+\frac{x}{\theta}\right)^{a}-1\right\}^{b}\right\}
$$

Then by inserting (2.2) and (6.2) in equation (6.1), we obtain

$$
\begin{aligned}
f_{i: n}(x)= & \sum_{m, h=0}^{\infty} b_{m+h+1} \frac{(m+h+1) a b}{\theta^{a}} x^{a-1}\left[\left(\frac{x}{\theta}\right)^{a}-1\right]^{b-1} \\
& \times \exp \left\{-(m+h+1)\left\{\left(\frac{x}{\theta}\right)^{a}-1\right\}^{b}\right\}
\end{aligned}
$$

where

$$
b_{k+h+1}=\frac{\alpha}{(m+h+1) \mathrm{B}(i, n-i+1)} \sum_{j=0}^{n-i}(-1)^{j+m+h}\left(\begin{array}{c}
n-i \\
j
\end{array}\right)\left(\begin{array}{c}
\alpha(i+j-1) \\
m
\end{array}\right)\left(\begin{array}{c}
\alpha-1 \\
h
\end{array}\right) .
$$

The $r$ th moment of $X_{i: n}$ (for $r<a$ ) follows from (6.3) as

$$
E\left(X_{i: n}^{r}\right)=\sum_{l, k, m, h=0}^{\infty} b_{m+h+1} V_{j, k}[(k+1) b+l] B\left(1-\frac{r}{a},[(k+1) b+l]\right)
$$

where

$$
V_{j, k}=\theta^{r} \frac{(-1)^{k}}{l ! k !} \frac{(m+h+1) b}{[(k+1) b+l]} \frac{\Gamma((k+1) b+l+1)}{\Gamma((k+1) b+1)} .
$$

The L-moments are analogous to the ordinary moments but can be estimated by linear combinations of order statistics. They exist whenever the mean of the distribution exists, even though some higher moments may not exist, and are relatively robust to the effects of outliers. Based upon the moments in equation (6.4), we can derive explicit expressions for the L-moments of $X$ as infinite weighted linear combinations of the means of suitable EWP distribution. They are linear functions 
of expected order statistics defined by

$$
\lambda_{r}=\frac{1}{r} \sum_{d=0}^{r-1}(-1)^{d}\left(\begin{array}{c}
r-1 \\
d
\end{array}\right) E\left(X_{r-d: r}\right), r \geq 1 .
$$

The first four L-moments are given by

$$
\begin{aligned}
& \lambda_{1}=E\left(X_{1: 1}\right), \\
& \lambda_{2}=\frac{1}{2} E\left(X_{2: 2}-X_{1: 2}\right), \\
& \lambda_{3}=\frac{1}{3} E\left(X_{3: 3}-2 X_{2: 3}+X_{1: 3}\right), \\
& \lambda_{4}=\frac{1}{4} E\left(X_{4: 4}-3 X_{3: 4}+3 X_{2: 4}-X_{1: 4}\right) .
\end{aligned}
$$

\section{Parameter Estimation}

In this section we discuss the method of maximum likelihood to estimate the parameters of the EWP distribution. Let $X_{1}, \ldots, X_{n}$ be a random sample from EWP distribution with unknown parameter vector $v=(a, b, \theta, \alpha)$. The likelihood function for $L$ is given by

$$
\begin{gathered}
L=\left(\frac{a b \alpha}{\theta^{a}}\right)^{n} \prod_{i=1}^{n}\left[x_{i}^{a-1}\left[\left(\frac{x_{i}}{\theta}\right)^{a}-1\right]^{b-1} \exp \left\{-\left[\left(\frac{x_{i}}{\theta}\right)^{a}-1\right]^{b}\right\}\right] \\
\prod_{i=1}^{n}\left[1-\exp \left\{-\left[\left(\frac{x_{i}}{\theta}\right)^{a}-1\right]^{b}\right\}\right]^{\alpha-1} .
\end{gathered}
$$

Let $s=\left(\frac{x}{\theta}\right)^{a}-1, p=1-e^{-s^{b}}$ and $z=\left(\frac{x}{\theta}\right)^{a} \ln \left(\frac{x}{\theta}\right)$.

Then the $\log$-likelihood function, $\ell=\ln L$, becomes:

$$
\begin{aligned}
\ell= & n \ln a+n \ln b+n \ln \alpha-n a \ln \theta+(a-1) \sum_{i=1}^{n} \ln x_{i} \\
& +(b-1) \sum_{i=1}^{n} \ln s_{i}-\sum_{i=1}^{n} s_{i}^{b}+(\alpha-1) \sum_{i=1}^{n} \ln p_{i} .
\end{aligned}
$$

Since $\theta$ is assumed known (as $x>\theta$ ), the score vector is $\mathbf{U}(v)=\frac{\partial \ell}{\partial v}=\left(\frac{\partial \ell}{\partial b}, \frac{\partial \ell}{\partial a}, \frac{\partial \ell}{\partial \alpha}\right)^{T}$. The elements of the score vector are given by

$$
\begin{aligned}
\frac{\partial \ell}{\partial b}= & \frac{n}{b}+\sum_{i=1}^{n} \ln s_{i}-\sum_{i=1}^{n} s_{i}^{b} \ln s_{i}+(\alpha-1) \sum_{i=1}^{n} \frac{s_{i}^{b} e^{-s_{i}^{b}} \ln s_{i}}{p_{i}}, \\
\frac{\partial \ell}{\partial a}= & \frac{n}{a}-n \ln \theta+\sum_{i=1}^{n} \ln x_{i}+(b-1) \sum_{i=1}^{n} \frac{z_{i}}{s_{i}} \\
& -b \sum_{i=1}^{n} s_{i}^{b-1}\left(\frac{x_{i}}{\theta}\right)^{a} \ln \left(\frac{x_{i}}{\theta}\right)+b(\alpha-1) \sum_{i=1}^{n} \frac{e^{-s_{i}^{b}} s_{i}^{b-1} z_{i}}{p_{i}}, \\
\frac{\partial \ell}{\partial \alpha}= & \frac{n}{\alpha}+\sum_{i=1}^{n} \ln p_{i} .
\end{aligned}
$$

We can find the estimates of the unknown parameters by setting the score vector to zero, $\mathbf{U}(\widehat{v})=0$, and solving them simultaneously yields the ML estimators $\widehat{a}, \widehat{b}$ and $\widehat{\alpha}$. These equations cannot be solved analytically and statistical software can be used to solve them numerically 
by means of iterative techniques such as the Newton-Raphson algorithm. For the four parameters EWP distribution all the second order derivatives exist.

For interval estimation of the model parameters, we require the $3 \times 3$ observed information matrix $J(v)=\left\{J_{r s}\right\}$ (for $\left.r, s=a, b, \alpha\right)$. Under standard regularity conditions, the multivariate normal $N_{3}\left(0, J(\widehat{v})^{-1}\right)$ distribution can be used to construct approximate confidence intervals for the model parameters. Here, $J(\widehat{v})$ is the total observed information matrix evaluated at $\widehat{v}$. Therefore, approximate $100(1-\phi) \%$ confidence intervals for $a, b$ and $\alpha$ can be determined as: $\widehat{a} \pm Z_{\frac{\phi}{2}} \sqrt{\widehat{J}_{a a}}$, $\widehat{b} \pm Z_{\frac{\phi}{2}} \sqrt{\widehat{J}_{b b}}$, and $\widehat{\alpha} \pm Z_{\frac{\phi}{2}} \sqrt{\widehat{J}_{\alpha \alpha}}$, where $Z_{\frac{\phi}{2}}$ is the upper $\phi$ th percentile of the standard normal distribution.

\section{Simulation Study}

In this section, we provide the simulation results to assess the performance of the proposed maximum likelihood estimation procedure. An ideal technique for simulating from (2.2) is the inversion method.

One would simulate $X$ by

$$
x=\theta\left\{1+\left[-\ln \left(1-u^{\frac{1}{\alpha}}\right)\right]^{\frac{1}{b}}\right\}^{\frac{1}{a}}, 0 \leq u \leq 1,
$$

where $U \sim U(0,1)$ is a uniform random number. For different combination of $a, b, \theta$ and $\alpha$ samples of sizes $n=100,200,300,500$ and 1000 are generated from the EWP distribution. We repeated the simulation $k=100$ times and calculated the mean and the root mean square errors (RMSEs). The empirical results are given in Table 1. It is evident that the estimates are quite stable and are close to the true value of the parameters for these sample sizes. Additionally, as the sample size increases the RMSEs, provided in the parentheses, decreases as expected.

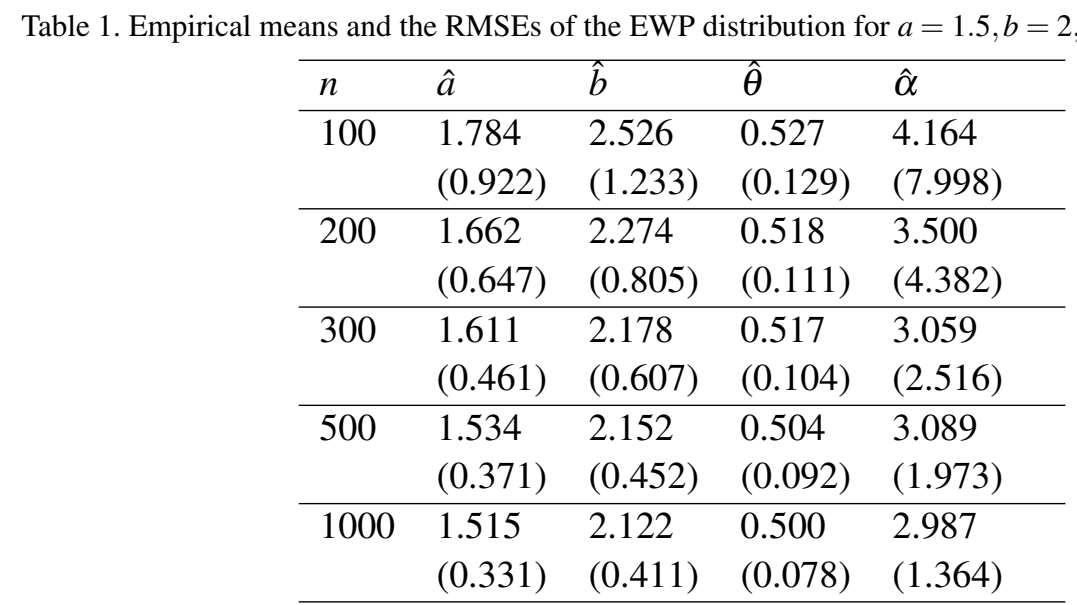

\section{Application}

In this section, we provide an application of the proposed EWP distribution to show its usefulness to fit real data set. The EWP model is compared with other related models, namely Weibull-Pareto 
(WP), Kumaraswamy Lomax (KwL) (Lemonte and Cordeiro [25]), transmuted Weibull Lomax (TWL) (Afify et al. [2]), McDonald Lomax (McL) (Lemonte and Cordeiro [25]), Weibull-Lomax (WL) (Tahir et al. [32]), transmuted complementary Weibull geometric (Afify et al. [1]), Pareto type IV (PIV)(Arnold [7]) and Lomax (L) distribution whose pdf's are given by

- The density of the KwL distribution is given by

$$
f(x)=\frac{\alpha a b}{\theta}\left(1+\frac{x}{\theta}\right)^{-(\alpha+1)}\left[1-\left(1+\frac{x}{\theta}\right)^{-\alpha}\right]^{a-1}\left\{1-\left[1-\left(1+\frac{x}{\theta}\right)^{-\alpha}\right]^{a}\right\}^{b-1},
$$

where $x>0, \alpha, \theta, a, b \geq 0$.

- The density of the TWL distribution is given by

$$
\begin{aligned}
f(x)= & \frac{a b \alpha}{\theta}\left(1+\frac{x}{\theta}\right)^{b \alpha-1}\left[1-\left(1+\frac{x}{\theta}\right)^{-\alpha}\right]^{b-1} \exp \left\{-a\left[\left(1+\frac{x}{\theta}\right)^{\alpha}-1\right]^{b}\right\} \\
& \times\left\{1-\gamma+2 \gamma \exp \left[-a\left[\left(1+\frac{x}{\theta}\right)^{\alpha}-1\right]^{b}\right]\right\},
\end{aligned}
$$

where $x>0, \alpha, \theta, a, b>0,|\gamma| \leq 1$.

- The density of the WL distribution is given by

$$
f(x)=\frac{a b \alpha}{\theta}\left(1+\frac{x}{\theta}\right)^{b \alpha-1}\left[1-\left(1+\frac{x}{\theta}\right)^{-\alpha}\right]^{b-1} \exp \left\{-a\left\{\left[\left(1+\frac{x}{\theta}\right)^{\alpha}-1\right]\right\}^{b}\right\},
$$

where $x>0, \alpha, \theta, a, b>0$.

- The density of the TCWG distribution is given by

$$
f(x)=\frac{\alpha \theta \gamma(\gamma x)^{\theta-1} e^{-(\gamma x)^{\theta}}\left[\alpha(1-b)-(\alpha-\alpha b-b-1) e^{-(\gamma x)^{\theta}}\right]}{\left(\alpha+(1-\alpha) e^{-(\gamma x)^{\theta}}\right)^{3}},
$$

where $x>0, \alpha, \theta, \gamma>0,|b| \leq 1$.

- The density of the McL distribution is given by

$$
f(x)=\frac{\alpha \gamma}{\theta B\left(a \gamma^{-1}, b\right)}\left(1+\frac{x}{\theta}\right)^{-(\alpha+1)}\left[1-\left(1+\frac{x}{\theta}\right)^{-\alpha}\right]^{a-1}\left\{1-\left[1-\left(1+\frac{x}{\theta}\right)^{-\alpha}\right]^{\gamma}\right\}^{b-1}
$$

where $x>0, \alpha, \theta, \gamma, a, b>0$.

- The density of the L distribution is given by

$$
f(x)=\frac{\alpha}{\theta}\left(1+\frac{x}{\theta}\right)^{-(\alpha+1)},
$$

where $x>0, \alpha, \theta>0$.

- The density of the PIV distribution is given by

$$
f(x)=\frac{\alpha}{\theta a}\left(\frac{x}{\theta}\right)^{\frac{1}{a}-1}\left[1+\left(\frac{x}{\theta}\right)^{\frac{1}{a}}\right]^{-\alpha-1},
$$

where $x>0, a, \alpha, \theta>0$. 


\section{A.Z. Afify et al.}

We analyze the data set which was originally reported by Badar and Priest [9] and referenced by several authors including Kundu et al. [24] and Afify et al. [3]. The data represent the strength, measured in GPa, for single carbon fibers and impregnated 1000-carbon fiber tows. Single fibers were tested under tension at gauge length $10 \mathrm{~mm}$. We estimate the unknown parameters of the EWP and other distributions discussed above using the method of maximum likelihood. Table 2 provides the MLEs of the model parameters and their standard errors (in parentheses).

Table 2. MLEs and their standard errors (in parentheses) for carbon fiber data set

\begin{tabular}{cccccc}
\hline \hline Model & \multicolumn{5}{c}{ Estimates } \\
\cline { 2 - 6 } & $\widehat{\alpha}$ & $\widehat{\theta}$ & $\widehat{a}$ & $\widehat{b}$ & $\widehat{\gamma}$ \\
\hline EWP & 43.773 & 0.5122 & 0.7787 & 1.329 & - \\
& $(101.835)$ & $(0.751)$ & $(1.498)$ & $(2.06)$ & - \\
WP & - & 0.0755 & 0.1834 & 13.9522 & - \\
& - & $(0.159)$ & $(0.102)$ & $(7.705)$ & - \\
PIV & 1545.411 & 654.611 & 0.7167 & - & - \\
& $(513.088)$ & $(183.946)$ & $(0.015)$ & - & - \\
TWL & 0.3922 & 0.6603 & 0.5287 & 8.4451 & 0.7364 \\
& $(0.339)$ & $(1.174)$ & $(3.32)$ & $(4.397)$ & $(0.286)$ \\
WL & 0.2417 & 0.3432 & 13.9237 & 10.0882 & - \\
& $(.373)$ & $(0.669)$ & $(269.788)$ & $(6.637)$ & - \\
McL & 45.9249 & 48.3024 & 18.1192 & 195.4633 & 353.1435 \\
& $(59.312)$ & $(63.047)$ & $(8.855)$ & $(123.217)$ & $(375.678)$ \\
TCWG & 0.2022 & 3.3482 & - & -0.0001 & 0.3876 \\
& $(0.217)$ & $(0.783)$ & - & $(0.496)$ & $(0.069)$ \\
KwL & 1.3863 & 0.545 & 60.4886 & 70.1112 & - \\
& $(0.553)$ & $(0.942)$ & $(100.286)$ & $(110.438)$ & - \\
L & 2184.8817 & 6683.0742 & - & - & - \\
& $(13139)$ & $(40191)$ & - & - & - \\
\hline \hline
\end{tabular}

The model selection is carried out using the AIC (Akaike information criterion), the BIC (Bayesian information criterion), the CAIC (consistent Akaike information criteria) and the HQIC (Hannan-Quinn information criterion). Note that the smaller the values of goodness-of-fit measures better the fit of the data. These measures are defined as

$$
\begin{aligned}
A I C & =-2 \ell(\hat{\Theta})+2 q \\
B I C & =-2 \ell(\hat{\Theta})+q \log (n) \\
H Q I C & =-2 \ell(\hat{\Theta})+2 q \log (\log (n)) \\
C A I C & =-2 \ell(\hat{\Theta})+\frac{2 q n}{n-q-1}
\end{aligned}
$$

where $\ell(\hat{\Theta})$ denotes the log-likelihood function evaluated at the maximum likelihood estimates, $q$ is the number of parameters, and $n$ is the sample size. Here $\Theta$ denote the parameters. Table 3 
lists the numerical values of the $-2 \ell(),. A I C, C A I C, H Q I C$ and $B I C$. These numerical results are obtained using the MATH-CAD PROGRAM.

Table 3. The statistics $-2 \ell$, AIC ,CAIC, HQIC and BIC for carbon fiber data set

\begin{tabular}{cccccc}
\hline \hline \multirow{2}{*}{ Model } & \multicolumn{5}{c}{ Goodness-of-fit criteria } \\
\cline { 2 - 6 } & $-2 \ell()$. & $A I C$ & $C A I C$ & $H Q I C$ & $B I C$ \\
\hline EWP & 112.874 & 120.874 & 121.563 & 124.245 & 129.446 \\
WP & 121.790 & 127.790 & 128.197 & 130.319 & 134.219 \\
KwL & 115.028 & 123.028 & 123.717 & 126.399 & 131.600 \\
TWL & 119.688 & 129.688 & 130.741 & 133.903 & 140.404 \\
WL & 121.749 & 129.749 & 130.439 & 133.121 & 138.322 \\
TCWG & 126.895 & 134.895 & 135.585 & 138.267 & 143.468 \\
McL & 130.597 & 140.597 & 141.65 & 144.812 & 151.313 \\
PIV & 228.386 & 234.386 & 234.793 & 236.915 & 240.815 \\
L & 266.925 & 270.925 & 271.125 & 272.611 & 275.211 \\
\hline \hline
\end{tabular}

From Table 3 it is evident that the EWP model has the lowest values for the AIC, CAIC, HQIC and BIC statistics among all fitted models. So, the EWP model could be chosen as the best fitted model for the subject data. The plots comparing the EWP distribution with other competing distribution is given in figure 3. These plots indicate that the EWP distribution fits the subject data well.

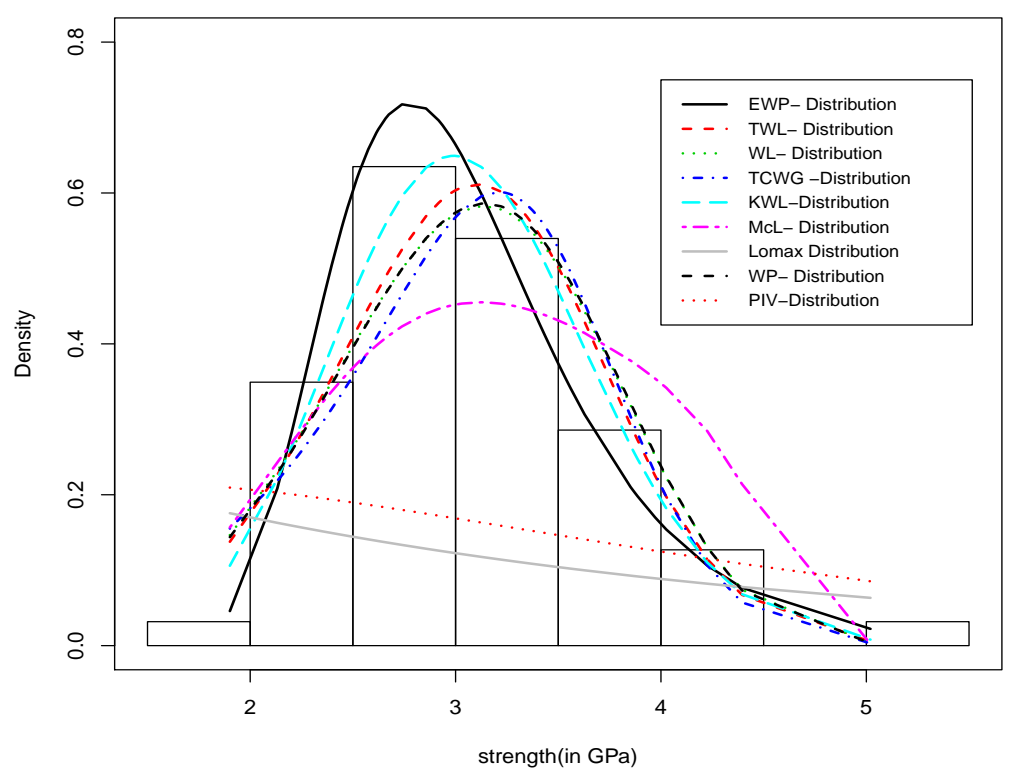

Fig. 3. fitted pdf of EWP and other distribution for the carbon fiber data 


\section{Conclusions}

In this paper, We propose a new four-parameter model, called the exponentiated Weibull-Pareto (EWP) distribution, which extends the Weibull- Pareto (WP) distribution introduced by Tahir et al. [33]. An obvious reason for generalizing a standard distribution is the fact that the generalization provides more flexibility to analyze real life data. We provide some of its mathematical properties. We derive explicit expressions for moments, generating function, Rényi and q-entropies . We also obtain the density function of the order statistics and their moments. We discuss maximum likelihood estimation. The proposed distribution is applied to a real data set. The EWP provides a better fit than several other nested and non-nested models. We hope that the proposed model will attract wider application in areas such as engineering, survival and lifetime data, meteorology, hydrology, economics and others.

\section{Acknowledgements}

The authors would like to thank the anonymous reviewers for their critical comments and suggestions which helped to improve the quality of the paper.

\section{References}

[1] A. Z. Afify, Z. M. Nofal and N. S. Butt, Transmuted complementary Weibull geometric distribution, Pak. j. stat.oper.res. 10(4) (2014) 435-454.

[2] A. Z. Afify, Z.M. Nofal, and A. N. Ebraheim, Exponentiated transmuted generalized Rayleigh distribution: a new four parameter Rayleigh distribution, Pak. j. stat. oper. res. 11 (2015) 115-134.

[3] A. Z. Afify, Z. M. Nofal, H. M. Yousof, Y. M. El Gebaly and N. S. Butt, The transmuted Weibull Lomax distribution: properties and application, Pak.j.stat.oper.res. 11 (2015) 135-152.

[4] A. Akinsete, F. Famoye, and C. Lee, The beta-Pareto distribution, Statistics 42(6) (2008) 547-563.

[5] A. Alzaatreh, F. Famoye and C. Lee, Weibull-Pareto distribution and its applications, Communications in Statistics-Theory and Methods 42 (2013) 1673-1691.

[6] A. Alzaatreh, F. Famoye and C. Lee, Gamma-Pareto distribution and its applications, Journal of Modern Applied Statistical Methods 11(1) (2012) 78-94.

[7] B. C. Arnold, Pareto Distributions, (International Cooperative Publishing House, Silver Spring, Maryland, 1983).

[8] G. Aryal and C. P. Tsokos, Transmuted Weibull Distribution: A Generalization of theWeibull Probability Distribution, European Journal of Pure and Applied Mathematics 4(2) (2011) 89-102.

[9] M. G. Badar and A. M. Priest, Statistical aspects of fiber and bundle strength in hydrid composites, In: Hayashi, T., Kawata, K., Umekawa, S. (Wds), progress in science and Engineering composites. ICCM-IV, Tokyo (1982) 1129-1136.

[10] M. Bourguignon, R. B. Silva and G. M. Cordeiro, The Weibull-G family of probability distributions, Journal of Data Science 12 (2014)53-68.

[11] M. Bourguignon, M. B. Silva, L. M. Zea and G. M. Cordeiro, The Kumaraswamy Pareto distribution, Journal of Statistical Theory and Applications 12 (2013) 129-144.

[12] G. M. Cordeiro, E. M. Ortega and S. Nadarajah, The Kumaraswamy Weibull distribution with application to failure data, Journal of the Franklin Institute 347(8) (2010) 1399-1429.

[13] F. Famoye, C. Lee and O. Olumolade, The beta-weibull distribution, J. Statist. Theor. Appl. 4(2) (2005) 121-136.

[14] W. Glänzel, A characterization theorem based on truncated moments and its application to some distribution families, Mathematical Statistics and Probability Theory (Bad Tatzmannsdorf, 1986), Vol. B, Reidel, Dordrecht (1987) 75-84. 
[15] W. Glänzel, Some consequences of a characterization theorem based on truncated moments, Statistics: A Journal of Theoretical and Applied Statistics, 21(4) (1990) 613-618.

[16] R. C. Gupta, P. L. Gupta and R. D. Gupta, Modeling failure time data by Lehmann alternatives, Communications in Statistics - Theory and Methods 27 (1998) 887-904.

[17] R. D. Gupta and D. Kundu, Generalized exponential distribution, Austral. \& New Zealand J. Statist. 41 (1999) 173-188.

[18] R. D. Gupta and D. Kundu, Generalized exponential distribution: different method of estimations, J. Statist. Comput. Simul. 00 (2000) 1-22.

[19] R. D. Gupta and D. Kundu, Exponentiated exponential family: an alternative to gamma and Weibull distributions, Biometrical J. 43 (2001) 117-130.

[20] R. D. Gupta and D. Kundu, Generalized exponential distribution: existing results and some recent developments, J. Statist. Plann. Inference 137 (2007) 3537-3547.

[21] G. G. Hamedani, Characterization of univariate continuous distribution, Technical report (2013).

[22] S. Huang and B. O. Oluyede, Exponentiated Kumaraswamy-Dagum Distribution with Applications to Income and Lifetime Data, Journal of Statistical Distributions and Applications 1(8) (2014).

[23] A. K. Kareema and M. A. Boshi, Exponential Pareto Distribution, Mathematical Theory and Modeling 3(5) (2013) 135-146.

[24] D. Kundu and M. Raqab, Estimation of $R=P(Y<X)$ for three-parameter Weibull distribution, Statistics and Probability Letters 79 (2009) 1839-1846.

[25] A. J. Lemonte and G. M. Cordeiro, An extended Lomax distribution, Statistics 47 (2013) 800-816.

[26] E. Mahmoudi, The beta generalized Pareto distribution with application to lifetime data, Mathematics and Computers in Simulation 81 (2011) 2414-2430.

[27] G. S. Mudholkar and G. D. Kollia, Generalized Weibull family: a structural analysis, Communications in statistics-theory and methods 23(4) (1994) 1149-1171.

[28] G. S. Mudholkar, D. K. Srivastava and M. Freimer, The expnentiated Weibull family: a reanalysis of the bus-motor-failure data, Technometrics 37(4) (1995) 436-445.

[29] S. Nadarajah, The exponentiated exponential distribution: a survey, AStA Advances in Statistical Analysis 95 (2011) 219-251.

[30] B. O. Oluyede and S. Rajasooriya, The Mc-Dagum distribution and its statistical properties with applications, Asian Journal of Mathematics and Applications (2013) 1-16.

[31] M. Shakil and M. Ahsanullah, Review on order statistics and record values from $F^{\alpha}$ distributions, Pak. J. Stat. Oper. Res. VIII (2012) 101-120.

[32] M. H. Tahir, G. M. Cordeiro, M. Mansoor and M. Zubair, The Weibull-Lomax distribution: properties and applications, The Hacettepe Journal of Mathematics and Statistics 44(2) (2015) 461-480.

[33] M. H. Tahir, G. M. Cordeiro, A. Alzaatreh, M. Mansoor and M. Zubair, A new Weibull-Pareto distribution: properties and applications, Communications in Statistics - Simulation and Computation $\mathbf{4 5}$ (2015) 3548-3567.

[34] L. M. Zea, R. B. Silva, M. Bourguignon. A. M. Santos and G. M. Cordeiro, The beta exponentiated Pareto distribution with application to bladder cancer susceptibility, International Journal of Statistics and Probability 1 (2012) 8-19. 